Review

\title{
Virtual Reality-Based Alcohol Prevention in Adolescents: A Systematic Review
}

\author{
Christina Prediger ${ }^{1, *}$, Stefanie Maria Helmer ${ }^{1}$, Robert Hrynyschyn ${ }^{1}$ (D) and Christiane Stock ${ }^{1,2}$ D \\ 1 Institute of Health and Nursing Science, Charité-Universitätsmedizin Berlin, Corporate Member of Freie \\ Universität Berlin and Humboldt-Universität zu Berlin, Augustenburger Platz 1, 13353 Berlin, Germany; \\ stefanie.helmer@charite.de (S.M.H.); robert.hrynyschyn@charite.de (R.H.); christiane.stock@charite.de (C.S.) \\ 2 Unit for Health Promotion Research, University of Southern Denmark, Niels Bohrs Vej 9, \\ 6700 Esbjerg, Denmark \\ * Correspondence: christina.prediger@charite.de; Tel.: +49-30-450-529-149
}

Citation: Prediger, C.; Helmer, S.M.; Hrynyschyn, R.; Stock, C. Virtual Reality-Based Alcohol Prevention in Adolescents: A Systematic Review. Adolescents 2021, 1, 138-150. https:// doi.org/10.3390/adolescents1020011

Academic Editor: Anne Campbell

Received: 26 March 2021

Accepted: 22 April 2021

Published: 28 April 2021

Publisher's Note: MDPI stays neutral with regard to jurisdictional claims in published maps and institutional affiliations.

Copyright: (c) 2021 by the authors. Licensee MDPI, Basel, Switzerland. This article is an open access article distributed under the terms and conditions of the Creative Commons Attribution (CC BY) license (https:/ / creativecommons.org/licenses/by/ $4.0 /)$.

\begin{abstract}
Virtual Reality-simulations offer new opportunities for alcohol prevention in adolescents. As an innovative medium, Virtual Reality can be attractive for the target group, and avatar-based pathways allow tailoring prevention in a gender-specific manner. However, tailoring may reproduce gender stereotypes and be exclusive. Therefore, this systematic review aims to summaries existing evidence regarding Virtual Reality-simulations for alcohol prevention targeting adolescents and to examine how gender is considered. A systematic search was conducted in seven databases. Two reviewers independently performed screening, data extraction, and quality assessment. Out of 224 search hits, four publications derived out of an Australian, a Danish, and a US-American project met the inclusion criteria. Research designs included qualitative (2), quantitative (1), and mixedmethod approaches (1). Qualitative methods focused on development, evaluation, and participatory methods in the creation process, demonstrating adolescents' involvement in Virtual Reality design. Adolescents appreciated the realism and the exposure to peer pressure and to negative alcohol consequences. Quantitative designs focused mainly on measuring alcohol-related outcomes (e.g., attitude and behavioral intentions towards binge drinking), and positive changes were found. In all studies, gender was assessed in the male-female dichotomy. Further gender-sensitive research is needed to develop and test the possibilities and pitfalls of tailoring Virtual Reality-simulations.
\end{abstract}

Keywords: virtual reality; alcohol prevention; adolescents; gender

\section{Introduction}

The harmful use of alcohol is linked with over 200 health conditions, such as injuries, cancer, and cardiovascular diseases [1]. Especially, adolescents tend to be vulnerable to alcohol-related risk factors. Alcohol use is associated with impairment in brain development [2], as well as with school problems, absenteeism, unwanted pregnancies, and the use of other substances [3,4]. Attitudes toward alcohol, drinking motives and consumption patterns are usually formed in adolescence [5]. Earlier initiation of consumption and regular use of alcohol during adolescence increases the likelihood of problematic consumption and dependence later in life $[3,5,6]$. Therefore, prevention plays a crucial role in adolescence.

Even though studies show that alcohol consumption among adolescents in European countries has been slightly but steadily declining in recent years [7], prevalence and highrisk behavior, such as binge drinking, remain high. Current data from 2019 shows that $79 \%$ of school students reported having consumed alcohol at least once during their life [7]. A total of $13 \%$ of the students reported having experienced alcohol intoxication in the last 30 days, and $34 \%$ stated at least one binge-drinking occasion (defined as five or more units of alcoholic beverages on one occasion) in the last month [7].

Virtual Reality (VR) is an innovative medium not only offering new treatment opportunities for psychological disorders [8], especially in craving provocation with substance 
use disorders [9], but also for alcohol prevention in adolescents. VR allows experiencing a specific situation in a virtual environment [10] by immersing in a simulation. These simulations can stimulate learning by a sense of presence and increased interactivity compared to traditional learning environments [11]. Moreover, VR allows experiences of risk situations in a safe environment [12] and thus to train skills securely. Further, the novelty of the medium can be attractive, especially to young people. Additionally, VR offers the possibility of creating different scenarios and simulation pathways according to game characters (avatars). Specifically tailored interventions in which the preventive strategy can be adapted to the individual's characteristics are promising tools for behavior change $[13,14]$. Therefore, the impact of VR on alcohol prevention should be analyzed.

One systematic literature review already summarized VR applications in individuals with alcohol misuse [15], alongside another review that provided an overview of the application of VR in alcohol studies in general [12]. The latter review showed the promising application of VR in various fields: (1) cue-exposure therapy, (2) driving simulators for examining relationships between alcohol and neurological activity, and (3) training of healthcare professionals. Only one of the included studies in this review focused on adolescents [12]. For that reason, the novelty of the present review is to focus on adolescents as a crucial target group of alcohol prevention. The impact of alcohol prevention using VR in adolescents offers opportunities for health improvement and should be analyzed.

Gender, as one of the central and complex social determinants of health [16], represents a possible customizable category for VR alcohol prevention. As differences between gender groups regarding the consumption and the social interaction with alcohol can be monitored $[6,7,17]$, and the effectiveness of prevention and health promotion depends on gender differences [18,19], it seems to be of significant importance to use the potential of tailoring VR alcohol prevention in a gender-sensitive manner. Moreover, gender can be used as a site of inquiry to focus on further diversity characteristics (sexual orientation, cultural or economic background, ability, etc.) when investigating possible tailoring categories. At the same time, tailoring approaches to gender may reproduce stereotypes and be exclusive. First, when creating for example a male and a female version, stereotype binary gender attributions could be represented and differences within gender groups could be inappropriately homogenized. Second, gender and sexual diversity could not be addressed at all, thus excluding all those who cannot or do not want to define themselves as male or female. This highlights the methodological dilemma of gender-sensitive research: Neither to reproduce binary gender attributions nor to abandon gender as a category of analysis of difference and specific intervention. Hence, it is of interest, how VR approaches considered gender. Gender is understood here as a complex [16,20], intersectional [21], and relational [22] psycho-socio-cultural variable with more than two possible expressions, including diversity as well as within-group heterogeneity $[23,24]$. Current research that addresses gender-sensitive approaches in health reports refers to a similar conceptualization of gender [22]. Therefore, the present review examines if and how gender was acknowledged in previous research as a secondary aim. Based on this background the present review aims to

(1) identify and summarize existing evidence regarding VR simulations for alcohol prevention targeting adolescents and

(2) examine if and how gender is taken into account in VR alcohol prevention approaches.

\section{Materials and Methods}

A systematic literature search was conducted in June 2020, according to the recommendations of the Preferred Reporting Items for Systematic Reviews and Meta-Analyses (PRISMA) checklist [25].

\subsection{Databases}

This systematic review deals with an interdisciplinary research question. Therefore, various scientific sub-disciplines and their relevant databases were integrated into the 
search. Seven databases covering the medical, psychological, and educational fields were searched: MEDLINE, Web of Science, Cochrane Library, PsychINFO, PSYNDEX, FIS Bildung Literaturdatenbank (German), and ERIC.

\subsection{Eligibility Criteria and Literature Search}

As not only interventional studies should be included in the review, the SPIDER framework (Sample, Phenomenon of Interest, Design, Evaluation, Research Type) was used to integrate quantitative, qualitative, and mixed-methods studies. SPIDER was used because it is particularly suitable for mixed-methods research [26]. For the Sample, adolescents between 10-19 years were selected according to WHO definition [27]. The search strategy also covered all common synonyms. VR served as the Phenomenon of Interest and it was included in the search with appropriate synonyms. As Design, all types of research studies and proposals were included. Our objective was not limited to report efficacy but to include all publications of this emerging research field. For Evaluation, we used the term alcohol prevent* and associated terms. As Research Type qualitative, quantitative, and mixed-methods studies were of interest. Therefore, a restriction was not set. Gender, as the particular interest of this review, was not applied as a search term to broaden the results.

Thus, the search terms result in the following search syntax: (adolescents OR young people OR youth OR young OR teenager OR underage OR minors) AND (virtual reality OR VR OR virtual simulation OR simulated 3D environment OR artificial reality) AND (alcohol prevent* OR alcohol* OR prevention of alcohol OR alcohol consumption OR alcohol misuse OR alcoholism OR binge drinking). Search terms were translated for the German database FIS. In addition, a hand search in included papers citations and reference lists was performed to explore further suitable publications.

\subsection{Selection of Studies}

An EndNote (X9.3.3) database was created to manage the search results. First, duplicates in search results were eliminated. Second, titles and abstracts were reviewed to determine whether the remaining studies meet the inclusion criteria. Inclusion criteria were: VR as intervention, intervention on alcohol (also among other scopes), only adolescents as target group, full text available, no grey literature (e.g., conference papers), and English or German language. Different publications that originated from the same study were included and assessed if they met the eligibility criteria. Two reviewers (C.P./S.M.H.) were involved in search results screening and checked all hits independently. If eligibility criteria were fulfilled, the two reviewers continued with reading the full texts of the corresponding articles. In case of a divergent decision on the eligibility of the two reviewers, studies were also included for full-text examination. During the eligibility checking of remaining full texts, reasons for exclusion were documented.

\subsection{Data Extraction}

Data extraction was performed independently by two reviewers (C.P./S.M.H.) using a standardized a priori extraction form consisting of the following segments: author, year, title, country, design, sample, objective, VR details, theory, outcomes measures, used instruments, results, discussion, limitations. In addition, publications were checked in detail on whether and how gender was acknowledged in the included studies. Results were saved in an Excel (version 2101) spreadsheet by each reviewer. A comparison of the two Excel sheets was used to check for accuracy of summaries or identify disagreements. Any discrepancies were resolved by referring to the original article, via discussion and subsequently led to a consented synthesized extraction database.

\subsection{Assessment of Study Quality}

Quality assessments served to identify consistent weaknesses across studies. Two reviewers (C.P./S.M.H.) independently conducted a critical evaluation of the study quality. 
The Mixed Method Appraisal Tool (MMAT), a quality assessment instrument for quantitative, qualitative, and mixed-methods studies [28], served as a basis for the assessment. The critical appraisal criteria were applied by each reviewer independently and then compared. Unclarity was resolved by discussion. Instructions by Hong et al. [28] were followed for extracting detailed information on the quality of each study instead of calculating an overall score, which allows a comprehensive and transparent overview. Quality assessments were not used to exclude any studies.

\subsection{Data Synthesis}

The results were analyzed and synthesized narratively, segregated by design, and orientated at a parallel-results convergent synthesis design as described by Noyes et al. [29] in numerical, tabular, or textual format. Conducting a meta-analysis was not foreseen since this review was not restricted to quantitative study designs.

\section{Results}

\subsection{Search Results}

The Database search retrieved 224 records. Most of the records were found in MEDLINE $(n=75)$, Cochrane Library $(n=62)$, and PsychINFO $(n=41)$. The search in Web of Science, PSYNDEX, and ERIC resulted in 29, 12, and 5 results, respectively. No suitable study was identified in the German database FIS. Hand search resulted in 11 additional publications. After duplicates were removed, 203 records were screened by reading titles and abstracts. Subsequently, 177 papers were excluded. Of the remaining 26 records, 22 were excluded due to the following reasons: Eleven studies were not dealing with the specified target group, seven records did not deal with VR as an intervention, two studies were not focusing on alcohol use and two studies did not provide a full text. Consequently, four records were included in the synthesis. The search process is visualized in Figure 1.

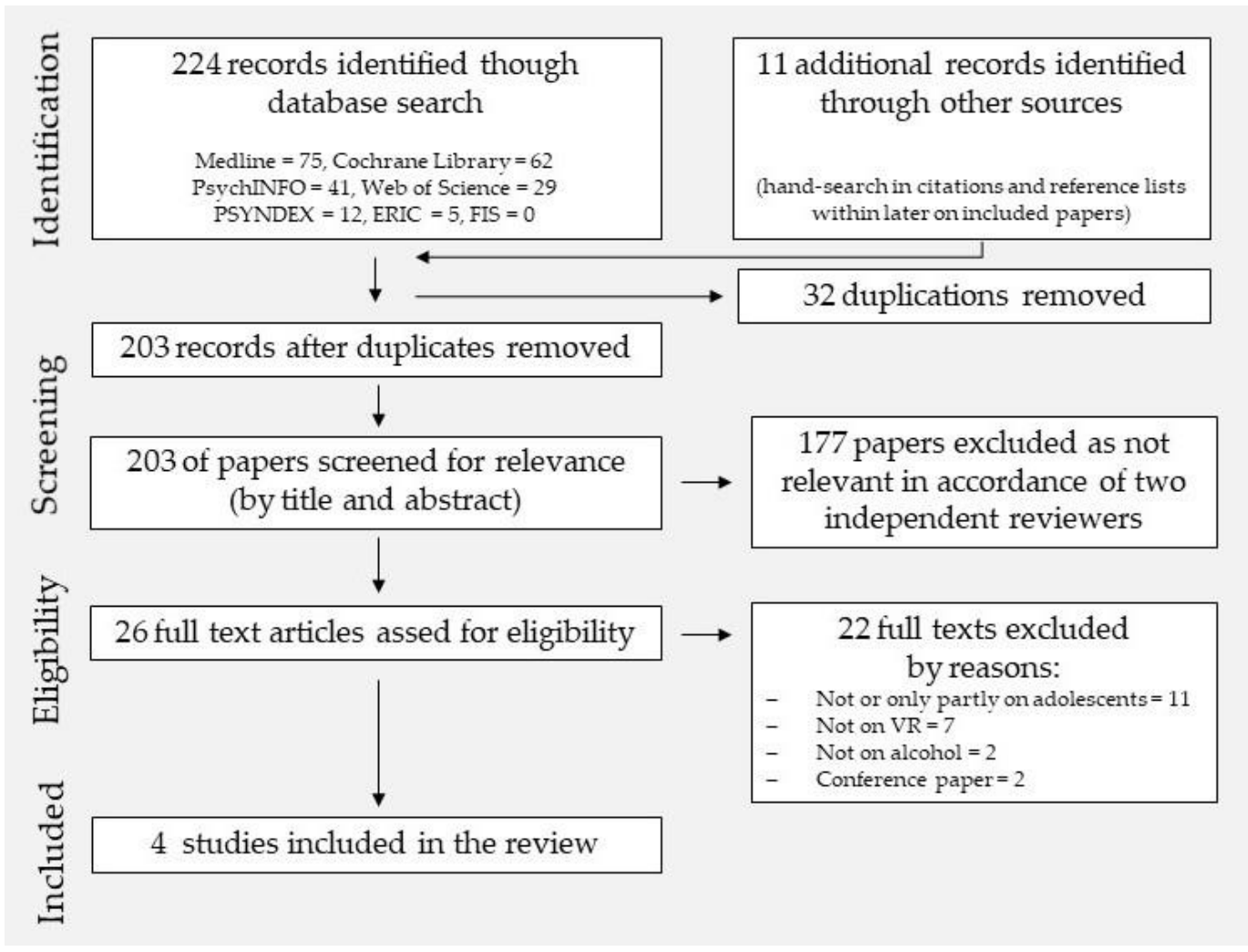

Figure 1. PRISMA flow diagram for the procedure of the systematic review. 


\subsection{Characteristics of the Studies}

All four included studies (see Table 1) were published after 2014 and derived out of an Australian [30,31], a Danish [32], and a US-American project [33]. The earliest publication provided information on an animated VR simulation whereas the other three studies were based on filmed VR simulations.

Two of the VR simulations were based upon an alcohol education program named $V R$ House Party as part of the Blurred Minds program [30,31]. This program featured a suite of gamified resources to engage and deliver alcohol-related messages to adolescents $[30,31]$. The VR House Party simulation was conceptualized "to experience peer pressure and drink on a no-risk (virtual) environment" [31] (p. 7). The Danish study used a revised version of the VR House Party film script. Based on that, the authors filmed a new VR simulation named VR FestLab [32]. Hadley et al. [33] created an animated VR simulation in which participants were exposed to alcohol and cannabis use and sexual risk-taking cues in a party environment for adolescents.

In all VR simulations, users experience different scenes (pre-party, party orientation, at the bar, etc.), and alcohol had been offered (in Hadley et al. [33] also marijuana). While Hadley et al. [33] provided a 7-min animation in which the participants could not make decisions themselves, VR House Party and VR FestLab were interactive set pathway simulations, and users could choose reactions and the scene they wanted to enter next.

The three studies based on VR House Party [30,31] or VR FestLab [32] aimed at similar objectives. The aims were the VR development or evaluation and how to apply participatory methods in the creation process. The objective of Hadley et al. [33] was to examine the utility of VR in eliciting psychological arousal by exposing participants to substance use and sexual risk-taking cues. According to different objectives, the designs varied. The methodology ranged from two qualitative studies [30,32], one quantitative evaluation [33] to one mixed-methods study [31]. Sample size substantially varied due to methodological approaches ( $n=9$ in qualitative interviews up to the assessment of pre-post quantitative data in the mixed-methods approach of 374 adolescents). Qualitative designs followed an exploratory approach of VR creation or evaluation, and adolescents' opinions and feelings towards the programs using various qualitative data sources. Qualitative data acquisition tools have been semi-structured face-to-face interviews with open-ended questions [32], written feedback forms [30,31], and protocolled observations as well as created presentations and generated ideas by participants of creation groups [30]. The quantitative study focusing on psychological arousal [33] measured changes in physiological outcomes on subjective and objective levels (heart rate, respiratory sinus arrhythmia, and self-reported somatic arousal) in a within-subject design by paper-pencil forms. The mixed-methods study [31] measured in the quantitative part changes in alcohol-related outcomes (e.g., attitude towards binge drinking or behavioral intentions) using a pre-post online survey. 
Table 1. Overview of study characteristics of included studies.

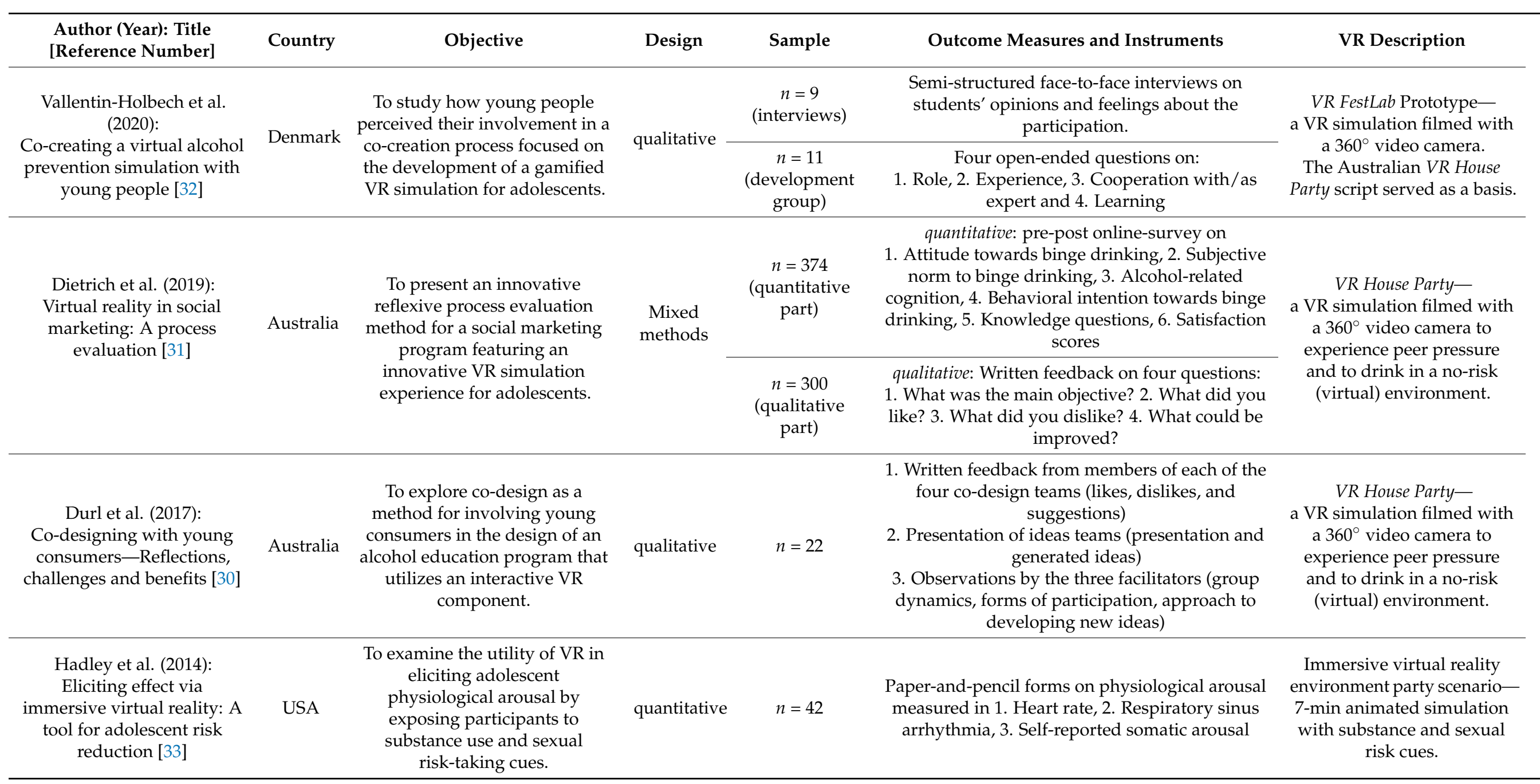




\subsection{Results of Assessment of Study Quality}

Overall, the quality of the studies was considered high based on the used MMAT checklist [28]. The study by Durl et al. [30] met the conditions in every category in the qualitative section and can be considered to be of high quality.

The study by Dietrich et al. [31] met most requirements regarding the mixed-methods category tool by both reviewers. However, the reviewers agreed on the lack of clarity regarding the qualitative results and quantitative methods. The qualitative findings were supported by only a few example quotes, which are a quality criterion for reporting qualitative research [34] to illustrate the analysis and conclusions in a transparent and convincing manner [35]. Nonetheless, this criterion was still sufficiently fulfilled, as it meets the scope of content analysis and the number of quotes is not defined in numbers [35]. In terms of the qualitative part of the mixed-methods, study information about the concrete sampling strategy was not reported. Furthermore, it did not become clear if the authors followed a strategy of dealing with divergences and inconsistencies between qualitative and quantitative findings.

The study by Vallentin-Holbech et al. [32] was considered to be of high quality in the category for qualitative studies as it met all conditions.

The study by Hadley et al. [33] was assessed based on the MMAT quantitative nonrandomized trial category. The participants seemed to be not representative of the target population and confounders were not accounted for in the design and analyses. However, the measurements were appropriate (subjective and objective), and the outcome data was rated as complete. Furthermore, the intervention was administered as intended.

\subsection{Study Findings}

Qualitative findings on the creation process demonstrated that adolescents or young people were intensively involved and influenced the participatory production of the VR simulation. They felt included in designing the VR simulation to a high degree and they were surprised that most of their ideas were integrated into the film script (e.g., mini games in VR FestLab) [32]. Participants reported benefits such as a feeling of courage, empowerment, self-confidence, and increased self-efficacy [32]. Durl et al. [30] likewise focused on the creation process. They highlighted the importance of sensitization and facilitation for active inclusion of the target group using interactive methods, such as role-plays.

Positive comments were made by the participants on the novelty and technical advantages of VR as a medium. The immersive experience, interactivity, control, and possibility to choose one's own path during the simulation have been mentioned [31,32]. "Realism" was often considered high [30,31]. Still, respondents requested improvements by further alignment of the story and contents with the realities faced by the target group [30].

Simultaneously, qualitative data revealed room for improvement and critical aspects. Quotes on the concept, technical, and film-related aspects, such as offering more options in decision making, the ability to move, improving the user interface, and optimizing the party setting or the actors were found [31]. A more accurate representation of the target group regarding the party setting, the music, characters, and language was further discussed [31]. The fact that peer pressure and negative consequences of alcohol drinking have been included in the simulations VR House Party and VR FestLab generally have been rated positively [31]. Nonetheless, participants of two studies stated that negative consequences could be more pronounced, which means more seriously or more frequently throughout the simulation and that peer pressure was not displayed in a sufficient intensity $[30,31]$. Moreover, qualitative approaches highlighted the importance of clarity about the scope of the participation and the objective of the simulation itself. In the study by Dietrich et al. [31] the main objective of the simulation (practicing decision making and experience consequences of drinking) was mostly understood by participants. However, some participants also mentioned divergent aims (avoiding or intending to get drunk). In line with these findings, Vallentin-Holbech et al. [32] noted that the overall purpose was 
not clear to all participants in the development group and that basic facts were not well understood by all such as that participation was voluntary, as well as information on the subsequent process was lacking for some of the participants.

Quantitative findings by Hadley et al. [33] and the quantitative approach in the mixed-methods study by Dietrich et al. [31] have been focusing on changes in associated measurements due to VR such as arousal by cues [33] or mainly on alcohol-related outcomes [31]. Alcohol-related outcomes in Dietrich et al. [31] showed changes on some of the applied scales. Regarding attitudes towards excessive drinking, a significant reduction was found and knowledge on alcohol significantly increased. Behavioral intention remained on a low level after experiencing the VR simulation. Furthermore, no change in social norms was found.

Satisfaction with the VR simulation and creation process was high, both in qualitative [32] and quantitative parts of the mixed-methods approach [31]. Dietrich et al. [31] compared the effect of satisfaction with the VR simulation on several outcome variables, showing an association with knowledge, drinking intentions, and attitudes towards binge drinking: Higher satisfied participants showed a higher increase in knowledge on alcohol compared to low satisfaction groups. Further, higher satisfied participants showed lower intention to drink excessively and more negative attitudes towards excessive drinking compared to the neutral satisfaction group.

Inconsistent results appeared on self-efficacy. While Vallentin-Holbech et al. [32] reported an increase in self-efficacy due to the involvement in the co-creation process, Dietrich et al. [31] found a reduction of self-efficacy on the applied "Drinking Refusal Self-Efficacy Questionnaire-Revised".

Hadley et al. [33] found a significant increase in heart rate and a significant decrease in respiratory sinus arrhythmia between neutral VR surroundings and a VR party simulation, indicating increasing physiological arousal. Differentiated results for the VR simulation segments (e.g., orientation/party introduction, substance use, and sexual risk) demonstrated that heart rate was significantly elevated across different scenes, whereas only the orientation/party introduction and sexual risk segments demonstrated a significant impact on respiratory sinus arrhythmia. Heart rate was elevated by substance use cues, while respiratory sinus arrhythmia was not.

In all four studies, gender was assessed in a dichotomous way (male-female). None of the studies outlined a definition or understanding of gender as a category. Each study reported an almost balanced gender proportion in their sample. The Australian project VR House Party and the US study [33] conceptualized a gender-specific simulation for boys and girls, while the Danish VR FestLab project did not outline gender-specific considerations. The US study was designed with opposite-sex flirting, while the other publications did not outline reflections on flirt orientation. In the developmental phase of the Australian project, differences occurred between the homogenous and mixed-gender groups. Homogeneous gender groups showed a greater degree of involvement than mixed groups, which could be due to a higher homogeneity of the group composition or higher sensitization incentives, as the authors acknowledge [30].

\section{Discussion}

Overall, our review revealed a modest research base showing the nascent nature of VR in alcohol prevention for adolescents. While the literature search was broad without restricting to specific research designs to identify all relevant publications in this field and to investigate the topic in its full complexity, results were rather sparse with only four suitable publications. Nonetheless, these studies revealed appealing findings due to diverse methodological approaches. Most of the studies focused primarily on developing and evaluating VR as a tool for alcohol prevention in adolescents. Two studies expressed the development of VR interventions. This reflects the early stage of research in this field.

The fact that "Realism" was considered to be a topic in all studies emphasizes the potential of VR. In addition, it was found that it is a challenge to develop a simulation that 
corresponds with the realities of the target group. High levels of involvement and influence in the co-designing process and positive feedback, such as on novelty and interactivity, are reflecting the advantages of VR as a prevention tool. This was especially true for the participatory creation process with the target group. Hadley et al. [33] developed the VR-animation through an iterative process based on the feedback of the target population that previously participated in a series of focus groups and showed involvement during the creation process. At the same time, qualitative findings demonstrate the further potential of improving technical and conceptual aspects. Technical aspects include VR and filmrelated features as user interface, decision options, or the ability to move. Advances in VR technology may point to the challenge of keeping up with user expectations and technical possibilities. On the conceptual side, peer pressure should play a more important role in VR prevention programs because reacting to peer pressure is one central educational goal. This is in accordance with the findings of Dalgaard Guldager et al. [36] who published a study on usability and game experience of VR FestLab after the systematic search of this review was conducted. Participants here pointed out that peer pressure in the VR simulation has been "less than what the adolescents might experience in real life" [36] (p. 9).

In general, clear information on the scope, the voluntariness of participation, the study process, and communication of results is not only an ethical necessity but crucial to the claims of participatory research and development [37] and thus cannot be stressed enough as study reports reflect (e.g., [32]).

Further, qualitative findings point to the necessity of a more accurate representation of the target group regarding party setting, characters, music, and language. As the target group is largely heterogeneous, this can result in a developmental challenge. Therefore, tailoring possibilities of VR, according to social categories, such as gender, could be promising. VR House Party [30,31] and the animation by Hadley et al. [33] have created a gender-specific version of their simulation, while gender issues have not been the subject of discussion in the publication of Vallentin-Holbech et al. [32]. In VR House Party, gender was used as a category to create gender-mixed and homogenous groups in the co-creation process. Following methodological literature [38-40], gender homogeneous groups can show more interaction as there is less need for a group to bond. Correspondingly, in Durl et al. [30] only-girl and only-boy groups showed higher levels of involvement. Still, the methodological assumption on group composition cannot be verified. Higher levels of involvement could also track back to higher sensitization incentives that were applied, as the authors acknowledge. In the study by Hadley et al. [33] flirting was reported to be conceptualized as opposite-sex, whereas the other publications did not mention considerations of sexual orientation. All studies reported an almost balanced gender proportion in their samples, but further gender-specific conceptualizations and analyses have not been outlined or conducted in none of the four included studies. Therefore, further gender considerations and research is needed. This is in accordance with Dir et al. [41] who demand to consider different risk factors between boys and girls for prevention and intervention on binge drinking among adolescents. As so far the possible or already implemented tailoring of VR simulations according to gender has not been reflected or outlined, future research should address the gender sensitivity of such approaches. Based on current research [22] this should be conducted by including both, a diversity- and intersectional understanding of gender. Quantitative analyses showed changes in alcohol-related scales and physiological arousal. Attitudes towards excessive drinking have been significantly reduced as well as knowledge has been significantly increased [31]. Significant increases in physiological arousal between a neutral virtual room and a virtual house party scenario were observed [33], thus serving as a promising base for building affect management skills practice. Meanwhile, behavioral intention towards binge drinking showed low maintenance, and social norms remained unchanged [31]. Taken together, these findings point to the potential of VR as an educational tool for alcohol prevention on an attitudinal and cognitive level. A change in intention towards binge drinking could not be observed. However, the findings need to be interpreted with caution as no randomized controlled trials were 
included in the review. Only within-subject study designs were conducted, and control conditions were missing. Furthermore, the findings do not allow any assumptions on the actual change of behavior. Evaluation studies are needed that combine methodological robustness with flexibility for varying VR conditions.

Satisfaction seems to play an important role in the creation process. User satisfaction was associated with knowledge, attitudes, and intention towards binge drinking, positively influencing these outcomes. Therefore, satisfaction with the intervention appears to be central and indicates that participants should be involved during the creation on a large scale.

The divergent outcomes on self-efficacy need to be reflected. While VallentinHolbech et al. [32] reported an increase in self-efficacy due to participation in the creation process, Dietrich et al. [31] measured an undesired reduction of self-efficacy. A measured reduction does not necessarily need to be counted as negative. It could also be a possible sign of increased awareness of the importance of peer pressure in which the programs intended to raise consciousness. These findings indicate that attention needs to be drawn to strengthening resistance skills with clear alternative behavior options which could be trained to ensure that the intervention does not lead to an unintended outcome.

Two studies used a pre-post design [31,33], while the other studies were cross-sectional, yet no longitudinal studies have been conducted. Therefore, effects on long-term behavioral change could not yet be assessed. This is in accordance with Durl et al. [12], Dietrich et al. [31], and Vallentin-Holbech et al. [32] who already have requested longitudinal and community-controlled trial designs effectiveness studies, as well as studies that compare VR and non-VR alternatives. In addition, as already Dietrich et al. [31] stated, the effectiveness of different story paths should be studied to ensure that all play-through possibilities are delivering the intended outcome, especially as VR simulations provide the potential of tailoring according to gender or other social categories.

This review has several limitations. Firstly, due to heterogeneous research activities resulting in diverse designs included in this review only a narrative synthesis according to Noyes et al. [29] was possible. Integration occurred in the interpretation of results in the discussion section. Nonetheless, the detailed description provided a profound insight into the recent developments of this emergent research field. Therefore, an emphasis on the creation process and first tests are a good basis for further research.

Secondly, this review is limited by its inclusion criteria. With seven databases of different disciplines, the preliminary approach was broad. Grey literature and conference papers have been excluded to give space to completed and peer-reviewed studies. As the research field is quickly developing the decision to include only peer-reviewed journal articles narrowed the findings. Therefore, ongoing research or current activities could not be detected. Further, the focus on adolescents led to an exclusion of eleven studies not focusing on this target group. It can be questioned if studies on young adults would have contributed to the review's objective. However, the possibilities of prevention programs during adolescence are age-specific according to different developmental periods [42]. Therefore, findings from young adults cannot directly be applied to adolescents as the specific target group of this review. In general, the total number of included studies was small. This might be due to the research field's novelty or to the specific research question of this review. Therefore conclusions have to be drawn carefully, as the data basis is sparse. However, for further development, results of comparable target groups, such as young or emerging adults, could be considered for developing VR as a prevention tool. In this regard, it can be rated as a good decision not to have included "gender" as a search term, as the number of results would have been even more limited.

Finally, the results of this review are restricted due to the limitations of the included studies. The qualitative findings [30-32] relate to their specific sample and design tasks and cannot be generalized. Also, the quantitative findings [31,33] lack control groups, which hinders a sufficient assessment of the efficacy or effectiveness of the program. In addition, the quantitative designs relied on self-reported measures and not on objective measure- 
ments concerning the behavior change of interest (e.g., alcohol use) itself. Nevertheless, all reported findings serve as a basis of this young research field and point to a promising application of VR in alcohol prevention among adolescents, which should be accompanied by further research.

\section{Conclusions}

This review summarizes the research using VR as an educational tool for alcohol prevention in adolescents. Findings indicate that evidence is sparse, as so far there are only a few ongoing projects, namely the Danish VR FestLab, the Australian VR House Party, and a US-American project by Hadley et al. [33]. Four publications, which were derived out of these projects, met the inclusion criteria with a clear emphasis on adolescent alcohol prevention. While the last-mentioned measured quantitatively psychological arousal by substance and sexual risk cues in an animated scenario, VR FestLab, and VR House Party aimed to build resistance skills among participants for peer pressure in a filmed VR party simulation. Publications here focused on diverse outcomes and have been applying both qualitative and mixed-methods designs. Findings demonstrate the strength of involving the target group during the creation process. Positive results have been reported on "likes", while other comments on "dislikes" reveal further development possibilities. Most importantly, peer pressure was recommended to be intensified, which is a crucial point, as resistance skills towards peer pressure are a central scope of the interventions.

Gender, assessed in all included studies in a dichotomous male-female expression, is partly considered during development and application, but not discussed or reported as a central aspect or possible category of customization - though two projects have developed gender-specific versions. Further research is needed to determine the potential, but also possible pitfalls that gender-specific versions may create.

As this research field is emerging, ongoing research can be expected. However, based on this review and based on the authors' research recommendations, further empirical investigation is needed-especially when using gender as a tailoring category in interventions.

Taken together, this systematic review suggests that studies in the field are limited in evaluating the effects of VR as a means of alcohol prevention. First promising results and further improvement possibilities warrant additional research. Gender-sensitive research is needed to develop and test the opportunities and challenges of tailoring VR simulations. Hence, cooperation with developers and researchers of previous projects as well as the target group can be promising. Based on this, innovative gender-sensible VR simulated interventions for alcohol prevention in young people should be developed and existing prevention programs need to be enhanced.

Author Contributions: Conceptualization, C.P., S.M.H., and C.S.; methodology, C.P.; identification C.P.; screening, eligibility, extraction, merging of extraction and quality assessment C.P. and S.M.H.; writing-original draft preparation, C.P.; writing—-review and editing, C.P., C.S., R.H., and S.M.H.; visualization, C.P.; supervision, C.S. All authors have read and agreed to the published version of the manuscript.

Funding: This research received no external funding.

Institutional Review Board Statement: Not applicable.

Informed Consent Statement: Not applicable.

Data Availability Statement: The data extraction spreadsheet is available on request.

Conflicts of Interest: The authors declare no conflict of interest. 


\section{References}

1. World Health Organisation. Global Status Report on Alcohol and Health 2018: World Health Organisation. 2019. Available online: https:/ /apps.who.int/iris/rest/bitstreams/1151838/retrieve (accessed on 18 February 2021).

2. Salmanzadeh, H.; Ahmadi-Soleimani, S.M.; Pachenari, N.; Azadi, M.; Halliwell, R.F.; Rubino, T.; Azizi, H. Adolescent drug exposure: A review of evidence for the development of persistent changes in brain function. Brain Res. Bull. 2020, 156, 105-117. [CrossRef]

3. Inchley, J.; Currie, D.; Vieno, A.; Torsheim, T.; Ferreira-Borges, C.; Weber, M.M. Adolescent Alcohol-Related Behaviours: Trends and Inequalities in the WHO European Region, 2002-2014; World Health Organisation Regional Office for Europe: Kopenhagen, Denmark, 2018.

4. Stolle, M.; Sack, P.-M.; Thomasius, R. Binge drinking in childhood and adolescence: Epidemiology, consequences, and interventions. Dtsch. Aerzteblatt Int. 2009, 106, 323-328. [CrossRef]

5. Kuntz, B.; Lange, C.; Lampert, T. Alkoholkonsum bei Jugendlichen-Aktuelle Ergebnisse und Trends. Gesundh. Kompakt 2015, 6, 1-12. [CrossRef]

6. Orth, B.; Merkel, C. Der Alkoholkonsum Jugendlicher und Junger Erwachsender in Deutschland. Ergebnisse des Alkoholsurveys 2018 und TRENDS. BZgA-Forschungsbericht; Bundeszentrale für Gesundheitliche Aufklärung: Köln, Germany, 2020. Available online: https:/ / www.bzga.de/fileadmin/user_upload/PDF/studien/Alkoholsurvey_2018_Alkohol-Bericht.pdf (accessed on 18 February 2021).

7. ESPAD Group. ESPAD Report 2019: Results from the European School Survey Project on Alcohol and Other Drugs Luxembourg. 2020. Available online: http:/ / www.espad.org/espad-report-2019\#downloadReport (accessed on 18 February 2021).

8. Freeman, D.; Reeve, S.; Robinson, A.; Ehlers, A.; Clark, D.; Spanlang, B.; Slater, M. Virtual reality in the assessment, understanding, and treatment of mental health disorders. Psychol. Med. 2017, 47, 2393-2400. [CrossRef] [PubMed]

9. Segawa, T.; Baudry, T.; Bourla, A.; Blanc, J.-V.; Peretti, C.-S.; Mouchabac, S.; Ferreri, F. Virtual Reality (VR) in assessment and treatment of addictive disorders: A systematic review. Front. Neurosci. 2020, 13, 1409. [CrossRef] [PubMed]

10. Slater, M. Place illusion and plausibility can lead to realistic behaviour in immersive virtual environments. Philos. Trans. R. Soc. Lond. B Biol. Sci. 2009, 364, 3549-3557. [CrossRef] [PubMed]

11. Hu-Au, E.; Lee, J. Virtual reality in education: A tool for learning in the experience age. Int. J. Innov. Educ. Res. 2018, 4, 215-226. [CrossRef]

12. Durl, J.; Dietrich, T.; Pang, B.; Potter, L.-E.; Carter, L. Utilising virtual reality in alcohol studies: A systematic review. Health Educ. J. 2018, 77, 212-225. [CrossRef]

13. Ryan, P.; Lauver, D.R. The efficacy of tailored interventions. J. Nurs. Scholarsh. 2002, 34, 331-337. [CrossRef]

14. Kreuter, M.W.; Oswald, D.L.; Bull, F.C.; Clark, E.M. Are tailored health education materials always more effective than non-tailored materials? Health Educ. Res. 2000, 15, 305-315. [CrossRef]

15. Ghiţă, A.; Gutiérrez-Maldonado, J. Applications of virtual reality in individuals with alcohol misuse: A systematic review. Addict. Behav. 2018, 81, 1-11. [CrossRef]

16. Davidson, K.W.; Trudeau, K.J.; van Roosmalen, E.; Stewart, M.; Kirkland, S. Perspective: Gender as a Health Determinant and Implications for Health Education. Health Educ. Behav. 2006, 33, 731-743. [CrossRef] [PubMed]

17. Erol, A.; Karpyak, V.M. Sex and gender-related differences in alcohol use and its consequences: Contemporary knowledge and future research considerations. Drug Alcohol Depend. 2015, 156, 1-13. [CrossRef] [PubMed]

18. Regitz-Zagrosek, V. Sex and gender differences in health. Science \& Society Series on Sex and Science. EMBO Rep. 2012, 13, 596-603. [CrossRef] [PubMed]

19. Östlin, P.; Eckermann, E.; Mishra, U.S.; Nkowane, M.; Wallstam, E. Gender and health promotion: A multisectoral policy approach. Health Promot. Int. 2006, 21 (Suppl. 1), 25-35. [CrossRef]

20. Tannenbaum, C.; Greaves, L.; Graham, I.D. Why sex and gender matter in implementation research. BMC Med. Res. Methodol. 2016, 16, 145. [CrossRef]

21. Bauer, G.R. Incorporating intersectionality theory into population health research methodology: Challenges and the potential to advance health equity. Soc. Sci. Med. 2014, 110, 10-17. [CrossRef]

22. Pöge, K.; Strasser, S.M.; Saß, A.-C.; Rommel, A. Civil society stakeholders' participation in national health reporting on sex/gender issues: A study protocol for an intersectionality-informed and sex/gender-sensitive approach to focus group research. BMJ Open 2020, 10, e033412. [CrossRef]

23. Rommel, A.; Pöge, K.; Krause, L.; Ludwig, S.; Prütz, F.; Saß, A.C.; Strasser, S.; Ziese, T. Geschlecht und Gesundheit in der Gesundheitsberichterstattung des Bundes. Konzepte und neue Herausforderungen. Public Health Forum 2019, $27,98-102$. [CrossRef]

24. Nielsen, M.W.; Stefanick, M.L.; Peragine, D.; Neilands, T.B.; Ioannidis, J.P.A.; Pilote, L.; Prochaska, J.J.; Cullen, M.R.; Einstein, G.; Klinge, I.; et al. Gender-related variables for health research. Biol. Sex Differ. 2021, 12, 23. [CrossRef]

25. Moher, D.; Liberati, A.; Tetzlaff, J.; Altman, D.G. Preferred reporting items for systematic reviews and meta-analyses: The PRISMA statement. PLoS Med. 2009, 6, e1000097. [CrossRef]

26. Cooke, A.; Smith, D.; Booth, A. Beyond PICO: The SPIDER tool for qualitative evidence synthesis. Qual. Health Res. 2012, 22, 1435-1443. [CrossRef] 
27. Dick, B.; Ferguson, B.J. Health for the world's adolescents: A second chance in the second decade. J. Adolesc. Health 2015, 56, 3-6. [CrossRef]

28. Hong, Q.N.; Pluye, P.; Fabregues, S.; Bartlett, G.; Boardman, F.; Cargo, M.; Dagenais, P.; Gagnon, M.P.; Griffiths, F.; Nicolau, B.; et al. Mixed Methods Appraisal Tool (MMAT), Version 2018. User Guide 2018. Available online: http: //mixedmethodsappraisaltoolpublic.pbworks.com/w/file/fetch/127916259/MMAT_2018_criteria-manual_2018-08-01_ENG. pdf (accessed on 18 February 2021).

29. Noyes, J.; Booth, A.; Moore, G.; Flemming, K.; Tunçalp, Ö.; Shakibazadeh, E. Synthesising quantitative and qualitative evidence to inform guidelines on complex interventions: Clarifying the purposes, designs and outlining some methods. BMJ Glob. Health 2019, 4 (Suppl. 1), e000893. [CrossRef] [PubMed]

30. Durl, J.; Trischler, J.; Dietrich, T. Co-designing with young consumers-Reflections, challenges and benefits. Young Consum. 2017, 18, 439-455. [CrossRef]

31. Dietrich, T.; Rundle-Thiele, S.; Kubacki, K.; Durl, J.; Gullo, M.; Arli, D.; Connor, J. Virtual reality in social marketing: A process evaluation. Mark. Intell. Plan. 2019, 37, 806-820. [CrossRef]

32. Vallentin-Holbech, L.; Dalgaard Guldager, J.; Dietrich, T.; Rundle-Thiele, S.; Majgaard, G.; Lyk, P.; Stock, C. Co-Creating a virtual alcohol prevention simulation with young people. Int. J. Environ. Res. Public Health 2020, 17, 1097. [CrossRef] [PubMed]

33. Hadley, W.; Houck, C.D.; Barker, D.H.; Garcia, A.M.; Spitalnick, J.S.; Curtis, V.; Roye, S.; Brown, L.K. Eliciting affect via immersive virtual reality: A tool for adolescent risk reduction. J. Pediatr. Psychol. 2014, 39, 358-368. [CrossRef]

34. O'Brien, B.C.; Harris, I.B.; Beckman, T.J.; Reed, D.A.; Cook, D.A. Standards for reporting qualitative research: A synthesis of recommendations. Acad. Med. 2014, 89, 1245-1251. [CrossRef]

35. Eldh, A.C.; Årestedt, L.; Berterö, C. Quotations in Qualitative Studies: Reflections on constituents, custom, and purpose. Int. J. Qual. Methods 2020, 19, 1609406920969268. [CrossRef]

36. Dalgaard Guldager, J.; Lavasani Kjær, S.; Lyk, P.; Dietrich, T.; Rundle-Thiele, S.; Majgaard, G.; Stock, C. User experiences with a virtual alcohol prevention simulation for danish adolescents. Int. J. Environ. Res. Public Health 2020, 17, 6945. [CrossRef] [PubMed]

37. Wright, M.T. Participatory health research: Origins and current trends. Bundesgesundh. Gesundh. Gesundh. 2021, 64, 140-145. [CrossRef] [PubMed]

38. Misoch, S. Qualitative Interviews; De Gruyter: Oldenburg, Germany, 2019.

39. Schulz, M.; Mack, B.; Renn, O. Fokusgruppen in der Empirischen Sozialwissenschaft: Von der Konzeption bis Zur Auswertung, 1st ed.; VS Verlag für Sozialwissenschaften: Wiesbaden, Germany, 2012.

40. McLafferty, I. Focus group interviews as a data collecting strategy. J. Adv. Nurs. 2004, 48, 187-194. [CrossRef]

41. Dir, A.L.; Bell, R.L.; Adams, Z.W.; Hulvershorn, L.A. Gender differences in risk factors for adolescent binge drinking and implications for intervention and prevention. Front. Psychiatry 2017, 8, 289. [CrossRef] [PubMed]

42. Onrust, S.A.; Otten, R.; Lammers, J.; Smit, F. School-based programmes to reduce and prevent substance use in different age groups: What works for whom? Systematic review and meta-regression analysis. Clin. Psychol. Rev. 2016, 44, 45-59. [CrossRef] 